\title{
Revisión
}

\section{La enfermedad renal crónica. Revisión de la literatura y experiencia local en una ciudad de Ecuador}

\author{
Brenda Lorena Pillajo Sánchez (DD $\square^{1,2}$, Juan Sebastián Guacho Guacho (D) 1,2, \\ e Iván Ricardo Moya Guerrero iD1 \\ ${ }^{1}$ Servicio de Medicina Interna, Hospital General Docente de Ambato, Ambato, Ecuador. \\ ${ }^{2}$ Facultad de Ciencias de la Salud, Universidad Técnica de Ambato, Ambato, Ecuador.
}

Cómo citar: Pillajo Sánchez BL, Guacho Guacho JS, Moya Guerrero IR. La enfermedad renal crónica. Revisión de la literatura y experiencia local en una ciudad de Ecuador. Rev. Colomb. Nefrol. 2021, 8(3), e396. https://doi.org/10.22265/acnef.8.3.396

Recibido:

03/Mar/2020

Aceptado:

17/Jun/2020

Publicado:

18/Ago/2021

\section{Resumen}

La enfermedad renal crónica (ERC) representa una problemática importante de salud pública a nivel mundial, pues tiene implicaciones clínicas y socioeconómicas significativas. Lo anterior ha motivado a incrementar los esfuerzos por parte de los sistemas sanitarios para mejorar su detección precoz, así como la coordinación entre las distintas especialidades médicas.

La evidencia científica ha demostrado que, en la actualidad, la incidencia de la ERC incrementa en correlación con el proceso de envejecimiento, siendo este uno de los factores asociados más importantes a tener en cuenta, junto con las enfermedades crónicas no transmisibles. Por lo tanto, se hace relevante realizar una revisión de la literatura disponible para exponer la situación actual de la ERC a nivel mundial y poderla comparar con la experiencia local de la ciudad de Ambato, Ecuador, esto desde un enfoque nefroprotector.

Palabras clave: enfermedad renal crónica, Ecuador, envejecimiento, geriatría, nefrología.

$\square$ Correspondencia: Hospital General Docente de Ambato. Avenida Unidad Nacional y Pasteur. Ambato, Ecuador. brenda.pillajo@hga.mspz3.gob.ec 


\title{
Chronic kidney disease. Literature review The local experience in an Ecuador city
}

\begin{abstract}
Chronic kidney disease (CKD) represents a major public health problem. It has significant clinical and socioeconomic implications. This disease increases the efforts of the health system for its early detection and to improve coordination between the different medical specialties. Scientific evidence has shown that the incidence of CKD increases in the population in correlation to the aging process and chronic non-transmissible diseases. Therefore, it is relevant to review the literature to expose the current situation of CKD worldwide and compare it with the local experience from Ambato, Ecuador, with a nephroprotective approach.
\end{abstract}

Keywords: chronic kidney disease, Ecuador, aging, geriatrics, nephrology.

\section{Introducción}

En definitiva, la enfermedad renal crónica (ERC) se ha convertido en un problema para la salud pública; esta se define como una anormalidad de la estructura o la función renal presente durante más de 3 meses y tiene implicaciones importantes para la salud. La ERC se clasifica según su etiología y el porcentaje del daño renal de acuerdo a la tasa de filtrado glomerular (G1 a G5); además, se le agrega una subcategoría por el grado de albuminuria (A1, A2, A3) dada su importancia como riesgo de enfermedad cardiovascular y gravedad de ERC (G1-5A1-A3) [1].

Los cambios a lo largo del tiempo en la prevalencia de la ERC son indiscutibles, pues en la actualidad cerca de 850 millones de personas son afectadas por esta enfermedad a nivel mundial; además, se estima que esta sea la quinta causa más común de muerte prematura para el año 2040.

La ERC también se considera un problema de salud pública debido a los altos costos que genera ya que cerca del 2-3\% del presupuesto anual de salud es consignado a diálisis y trasplante renal en los países de ingresos altos [2].

\section{ERC a nivel mundial}

A pesar de su alta heterogeneidad, el primer metaanálisis sobre la prevalencia de ERC a nivel mundial que proporcionó una visión general exhaustiva de la literatura reportó cifras de 13,4\% para las etapas G1 a G5 y de 10,6\% para las etapas G3 a G5, además indicó 
que en los estadios finales de la enfermedad (G4 y G5) el porcentaje de pacientes es menor según reportes de Europa, Japón y Estados Unidos [3]. En 2017 casi 1,2 millones de personas murieron por ERC en todo el mundo y la tasa global de mortalidad de todas las edades por esta causa aumentó un 41,5\% (IC95\%: 35,2-46,5) entre 1990 y 2017, aunque no hubo cambios significativos en la tasa de mortalidad estandarizada por edad [4].

Para el año 2017 también se registraron 697,5 millones (IC95\%: 649,2-752,0) de casos de ERC en todas las etapas, lo que arrojó una prevalencia global de 9,1\%. Por otra parte, la prevalencia global de ERC en todas las edades aumentó 29,3\% (IC95 \%: 26,4-32,6) desde 1990, mientras que la prevalencia estandarizada por edad se mantuvo estable. En varias regiones, particularmente Oceanía, África subsahariana y América Latina, la carga de ERC fue mucho mayor de lo esperado para el nivel de desarrollo, mientras que la tasa de la enfermedad en África subsahariana occidental, oriental y central, Asia oriental, sur de Asia, Europa central y oriental, Australasia oriental y La enfermedad renal crónica (ERC) se ha convertido en un problema para la salud pública Europa occidental fue menor a loesperado [4].

La prevalencia de ERC en España supera el $20 \%$ en mayores de 60 años y aumenta hasta $40 \%$ en los pacientes octogenarios con otras comorbilidades [5], mientras que en Estados Unidos, según la CDC [6], fue del $7 \%$ en adultos entre 18 a 44 años en el 2019, lo que equivale a 37 millones de personas; en este país la ERC también es más común en adultos mayores (38\%) que en adultos entre 45 y 64 años $(13 \%)$.

\section{ERC en Latinoamérica}

Según la Sociedad Latinoamericana de Nefrología e Hipertensión (SLANH) y la Organización Panamericana de la Salud (OPS), citados por el Ministerio de Salud Pública de Ecuador [7], para el año 2013 la prevalencia de la enfermedad renal en Latinoamérica fue de 650 pacientes por millón de habitantes y registró un incremento estimado del $10 \%$ anual. Para el mismo año, Gámez-Jiménez et al. [8] indicaron que en la región la prevalencia fue de 447 pacientes por millón de habitantes, mientras que la incidencia correspondió a 147 pacientes por millón de habitantes; estos autores también indican que la distribución según países es de 1.026 pacientes por millón de habitantes en Puerto Rico, 667 pacientes por millón de habitantes en Argentina, 485 pacientes por millón de habitantes en Brasil y 63 pacientes por millón de habitantes en Bolivia. 


\section{ERC en Ecuador}

Para 2015 Ecuador contaba con 16.278.844 habitantes, y de estos se estima que 11.460 padecían ERC, alcanzando una mortalidad de 6-7\%; además, esta enfermedad generó un costo por diálisis de 168.342.720 dólares. La ERC también es la cuarta causa de mortalidad general y la quinta de mortalidad prematura en el país y produce el 1,44\% de años vividos con discapacidad [9].

Tomando en cuenta las estimaciones de la SLANH y de la Tercera Encuesta de Salud y Nutrición, en Ecuador se estima que aproximadamente el $45 \%$ de los pacientes con ERC en estadios IV y V podrían fallecer antes de iniciar el tratamiento con diálisis [9].

La ERC es una entidad clínica bien identificada que puede tener muchas causas asociadas a factores de riesgo comunes a otras enfermedades crónicas no trasmisibles. Así, en el mundo, 1,4 millones de muertes están relacionadas con enfermedades cardiovasculares y 25,3 millones de casos de enfermedades cardiovasculares fueron atribuibles a insuficiencia renal [4]. En general, el $30 \%$ de los casos de ERC se debe a causas relacionadas a diabetes mellitus; el $25 \%$, a hipertensión arterial, y el $20 \%$, a glomerulopatías [10].

\section{ERC en Ambato}

En Ecuador no existen estudios sobre factores de progresión de ERC en el grupo poblacional mayor de 65 años, además solo se reportan cifras en relación a los pacientes que reciben terapia de sustitución renal sin reflejarse las etapas previas de ERC, por lo que a continuación se detalla la situación de un hospital de segundo nivel de atención de salud de la ciudad de Ambato desde unaperspectiva retrospectiva y transversal.

Entre el 2017 y 2019 se atendieron 3.007 pacientes con diagnóstico de ERC (862 en 2017, 956 en 2018 y 1.189 en 2019). La distribución entre mujeres y hombres fue de $55 \%$ vs. $45 \%$ para $2017,47 \%$ vs. $53 \%$ para 2018 y $52 \%$ vs. $48 \%$ para 2019 (figura 1), y en todo el periodo de estudio, el $55 \%$ tenía más de 65 años, el $43 \%$ tenía entre 18 y 64 años y el $2 \%$ eran menores de 18 años (figura 2); además, el $74 \%$ residía en el área urbana y el $26 \%$, en el área rural (figura 3). La clasificación de ERC según los estadios se muestra en la figura 4.

\section{Nefroprotección}

La OPS señala que la ERC, aunque se puede prevenir, no tiene cura y es progresiva, silenciosa y asintomática hasta etapas avanzadas; además, su terapéutica (diálisis y trasplante renal) es invasiva y costosa [11]. En este contexto, muchos países carecen de recursos suficientes con 


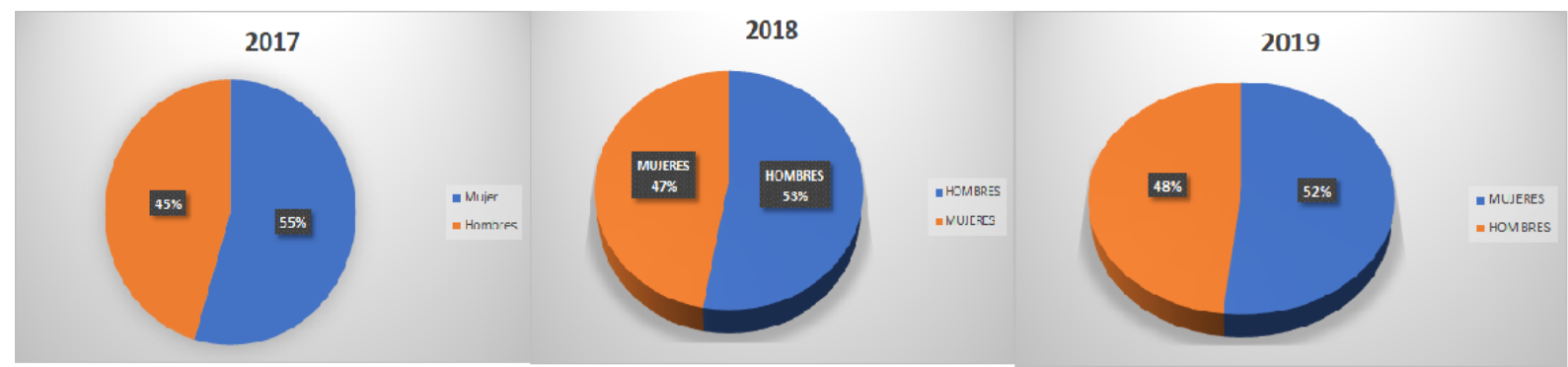

Figura 1: Distribución por género de pacientes con enfermedad renal crónica en Ambato, Ecuador, entre 2017 y 2019.

Fuente: elaboración propia.

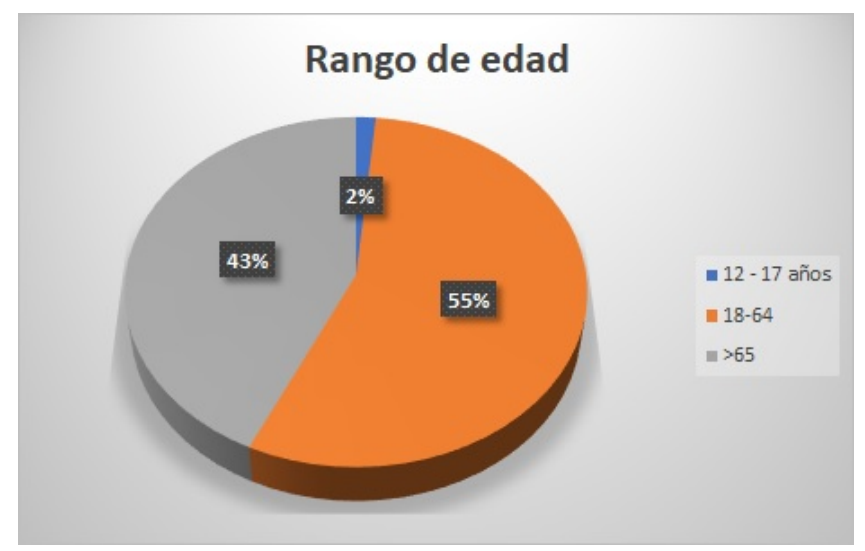

Figura 2: Distribución por edad de pacientes con enfermedad renal crónica en Ambato, Ecuador, entre 2017 y 2019.

Fuente: elaboración propia.

los cuales adquirir los equipos necesarios para brindar los tratamientos indicados a las personas que padecen esta enfermedad y la cantidad de especialistas disponibles en estas naciones también es insuficiente [11]. En síntesis, la ERC constituye un estadodefinitivo e incurable, y por eso la importancia de tomar medidas preventivas antes de su desarrollo.

Cuando un paciente en estadios finales de ERC no ingresa a tratamiento dialítico su expectativa de vida se reduce sustancialmente [12]; por lo tanto, se debe dar un cambio de enfoque, pasando del curativo al preventivo, y es a partir de esto que se propone la nefroprotección en tres fases:

\section{Fase primaria}

Esta fase incluye los individuos que aún no tienen la enfermedad y se enfoca en detectar y manejar factores de riesgo para ERC implementando medidas educativas en la población 


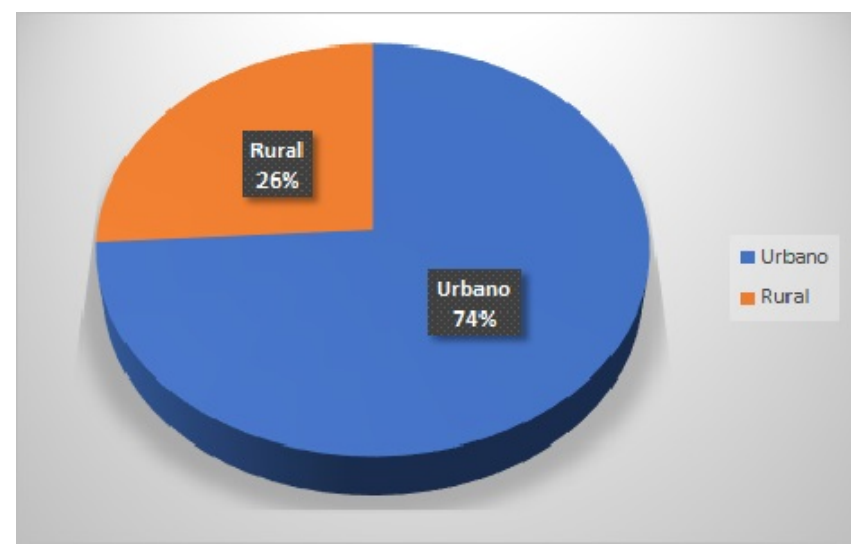

Figura 3: Distribución por lugar de residencia de pacientes con enfermedad renal crónica en Ambato, Ecuador, entre 2017 y 2019.

Fuente: elaboración propia.

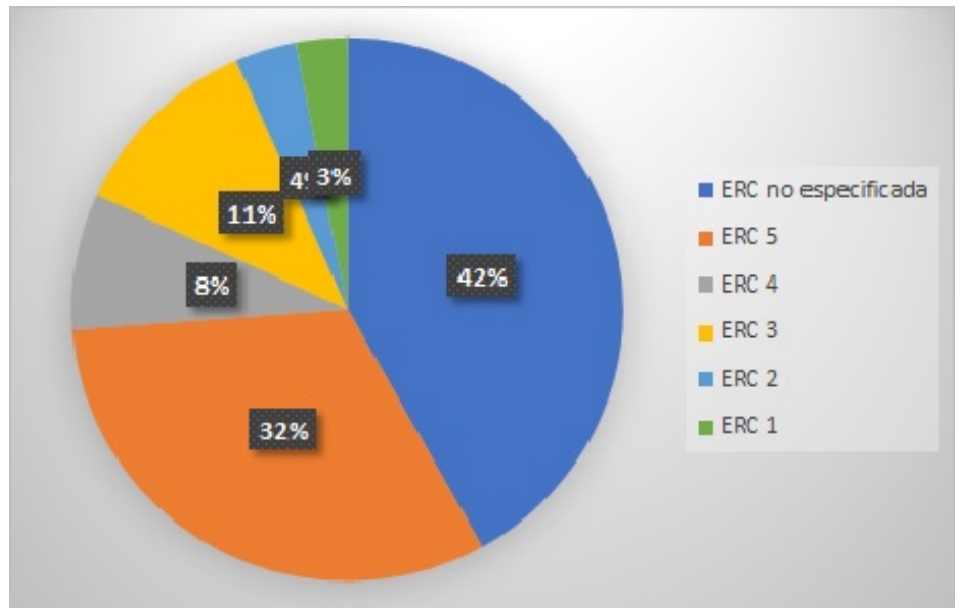

Figura 4: Distribución según clasificación del estadio de la enfermedad renal crónica en pacientes de Ambato, Ecuador, entre 2017 y 2019.

Fuente: elaboración propia.

general. Los principales factores de riesgos para desarrollar ERC son diabetes mellitus e hipertensión arterial, por lo que se deben alcanzar controles glicémicos y de presión arterial adecuados [12]. De igual forma, es necesario tener en cuenta otros factores de riesgo para ERC como obesidad, patologías cardiacas, antecedente familiar de ERC, edad avanzada y daño renal previo [6].

Los puntos importantes en este tipo de prevención son la intervención en factores modificables como los estilos de vida saludable: realizar actividad física regular, controlar la obesidad, dejar de fumar y mantener una dieta baja en sodio y proteínas. También es indispensable man- 
tener una adecuada hidratación y tener cuidado con condiciones especiales como pacientes monorrenos, enfermedad poliquística del adulto, etc. [12].

\section{Fase secundaria}

Debido a que los estadios iniciales de la ERC son asintomáticos, en esta fase la prevención busca detectar a quienes tienen la enfermedad, para lo cual se sugiere tamizar ciertas alteraciones en exámenes de laboratorio como microalbuminuria, hematuria y elevación de los niveles de creatinina y cistatina C. Esta fase es determinante ya que el estudio AusHEART demostró que solo el $18 \%$ de individuos con alteraciones de la función renal fueron correctamente diagnosticados de ERC [13].

Las intervenciones que se realizan en esta segunda fase pretenden disminuir la progresión de la ERC mediante dos pilares fundamentales: la reducción de la severidad de la proteinuria y la disminución de la presión intraglomerular, lo cual se logra implementando medidas farmacológicas y dietéticas [12]. En un estudio con 1.312 participantes, Chen \& Harris [13] evidenciaron que solo en el $50 \%$ se prescribió tratamiento para hipertensión arterial de forma adecuada, lo cual hace prioritario educar a los médicos de atención primaria en el manejo de la patología renal.

\section{Fase terciaria}

La prevención terciaria se enfoca en pacientes que ya tienen estadios avanzados de ERC y sus objetivos son retrasar el inicio de la diálisis y prevenir el desarrollo de lesión renal aguda, ya que la superposición de las dos conllevaría a la necesidad de diálisis emergente, lo que a su vez implicaría mayor incidencia deriesgo de hipotensión, deterioro de la función cardiaca, síndrome cardiorenal y retención hídrica.

En los pacientes con ERC se deben evitar los nefrotóxicos (antiinflamatorios no esferoidales, algunos antibióticos, suplementos herbales y contrastes endovenosos) [6]. Además, es importante que se haga un control adecuado de anemia, desordenes del metabolismo mineralóseo, acidosis metabólica e hipercalemia, así como un manejo conservador de la uremia [12].

\section{Conclusiones}

La ERC es un problema de salud pública a nivel global debido a las complicaciones que implica, tanto por su epidemiología, como por los costos elevados que acarrea y la multi morbimortalidad que ocasiona. Generalmente, al ser una entidad que inicialmente no causa 
síntomas, no es detectada de manera precoz, lo que tiene consecuencias negativas a largo plazo y por lo que es indispensable levantar líneas de base en cada localidad para realizar intervenciones oportunas y sobre todo aplicar las medidas de nefroprotección pertinentes.

La presente investigación corrobora muchos de los aspectos ya conocidos de la ERC a nivel mundial en cuanto a la etiología, la edad de presentación y el porcentaje de prevalencia de acuerdo al estadio de la ERC, por lo que se evidencia la necesidad urgente de realizar y desarrollar programas de nefroprotección renal en Ambato como única medida para evitar que el sistema desalud colapse debido al ingreso masivo de pacientes en estadios G4-G5 que requieren terapia de sustitución renal.

\section{Consideraciones éticas}

Los autores declaran que los procedimientos seguidos se realizaron conforme a las normas éticas del comité de experimentación humana responsable y de acuerdo con lo establecido por la Asociación Médica Mundial en la Declaración de Helsinki; que han seguido los protocolos de su centro de trabajo sobre la publicación de datos de pacientes, y que han obtenido el consentimiento informado de los pacientes y/o sujetos referidos en el artículo.

\section{Conflicto de intereses}

Ninguno declarado por los autores.

\section{Financiación}

Ninguna declarada por los autores.

\section{Contribución de los autores}

Brenda Pillajo: conceptualización y diseño de la investigación, interpretación de los datos y redacción, revisión y edición del manuscrito. Sebastián Guacho: aporte de pacientes y revisión y edición del manuscrito. Ricardo Moya: recolección y obtención de resultados y edición del manuscrito.

\section{Referencias}

[1] Kdigo Disease: Improving Global Outcomes (KDIGO) CKD-MBD Update Work Group. KDIGO 2017 clinical practice guideline updatefor the diagnosis, evaluation, prevention, 
andtreatment of chronic kidney disease-mineral andbone disorder (CKD-MBD). Kidney Int Suppl. 2017;7(1):1-59. https:/dx.doi.org/10.1016/j.kisu.2017.04.001. $\uparrow$ Ver página 2

[2] World Kidney Day. 14 de marzo de 2020. 2020 [citado abril 15 2020]. Disponible en: https: //www.worldkidneyday.org/ckd-2020-spanish/. ^Ver página 2

[3] Hill NR, Fatoba ST, Oke JL, Hirst JA, O’Callaghan CA, Lasserson DS, et al. Global Prevalence of Chronic Kidney Disease - A Systematic Review and Meta- Analysis. PloS One. 2016;11(7):e0158765. https:/doi.org/10.1371/journal.pone.0158765. 个Ver página 3

[4] GBD Chronic Kidney Disease Collaboration. Global, regional, and national burden of chronic kidney disease, 1990-2017: a systematic analysis for the Global Burden of Disease Study 2017. Lancet. 2020;395(10225):709-33. https://doi.org/10.1016/S0140-6736(20)30045-3. 个Ver página 3, 4

[5] Mora-Gutiérrez JM,Slon-Roblero MF, Castaño-Bilbao I, Izquierdo-Bautista D, ArteagaColoma J, Martínez-Velilla N. Enfermedad renal crónica en el paciente anciano.Rev Esp Geriatr Gerontol. 2017;52(3):152-8. https://doi.org/10.1016/j.regg.2016.03.006. 个Ver página3

[6] Centers for Disease Control and Prevention (CDC). Chronic Kidney Disease in the United States, 2019. Atlanta, GA: US Department of Health and Human Services, Centers for Disease Control and Prevention; 2019. $\uparrow$ Ver página 3, 6, 7

[7] Ecuador. Viceministerio de Atención Integral en Salud, Dirección Nacional de Centros Especializados. Programa Nacional de salud renal. Quito: Ministerio de Salud Püblica; 2015. 个Ver página 3

[8] Gámez-Jiménez AM, Montell-Hernández OA, Ruano-Quintero V, Alfonso de León J. Enfermedad renal crónica en el adulto mayor. Rev. Med. Electrón. 2013;35(4):306-18. $\uparrow$ Ver página 3

[9] Ecuador. Ministerio de Salud Pública (MSP). Prevención, diagnóstico y tratamiento de la enfermedad renal crónica. Guía de práctica clínica. Quito: MSP; 2018 [citado abril 15 2020]. Disponible en: https:/www.salud.gob.ec/wp-content/uploads/2018/10/ guia_prevencion_diagnostico_tratamiento_enfermedad_renal_cronica_2018.pdf. 个Ver página 4

[10] Rico-Fontalvo JE. Enfermedad renal diabética: de cara a la prevención, diagnóstico e intervención temprana. Rev Colomb Nefrol. 2020;7(2). https:/doi.org/10.22265/acnef.7.2.506. 个Ver página 4

[11] Organización Panamericana de la Salud (OPS).La OPS/OMS y la Sociedad Latinoamericana de Nefrología llaman a prevenir la enfermedad renal y a mejorar 
el acceso al tratamiento. Ginebra: OPS; 2015 [citado abril 15 2021]. Disponible en: $\quad$ https:/www.paho.org/hq/index.php?option=com_content\&view=article\&id=10542: 2015-opsoms-sociedad-latinoamericana-nefrologia-enfermedad-renal-mejorar-tratamiento\& Itemid=1926\&lang=es. $\uparrow$ Ver página 4, 5

[12] Kalantar-Zadeh, Li PK. Strategies to prevent kidney disease and its progression. Nat Rev Nephrol. 2020;16(3):129-30. https://dx.doi.org/10.1038/s41581-020-0253-1. 个Ver página 5, 6, 7

[13] Chen T, Harris D. Challenges of chronic kidney disease prevention. Med J Aust. 2015;203(5):209-10. https://dx.doi.org/10.5694/mja15.00241. 个Ver página 7 